Bull. Austral. Math. Soc.

Vol. 39 (1989) [25-30]

\title{
JAMES QUASI REFLEXIVE SPACE HAS THE FIXED POINT PROPERTY
}

\begin{abstract}
M.A. KHAMSI
We prove that the classical sequence James space has the fixed point property. This gives an example of Banach space with a non-unconditional basis where the Maurey-Lin's method applies.
\end{abstract}

\section{INTRODUCTION}

Let $K$ be a nonempty weakly compact convex subset of a Banach space $X$. We say that $K$ has the fixed point property (f.p.p.) if every non-expansive mapping $T: K \rightarrow K$ (that is $\|T(x)-T(y)\| \leqslant\|x-y\|$ for any $x, y$ in $K$ ) has a fixed point. We say that $X$ has the fixed point property (f.p.p.) if every weakly convex compact subset of $X$ has the f.p.p.

A theorem of Kirk [9] states that if $K$ has normal structure, then it has the f.p.p. It was unknown whether the normal structure is essential. Karlovitz [7] answered the problem negatively.

Alspach [1] proved that $L_{1}$ fails the f.p.p., proving that weak compactness is not sufficient to have the f.p.p. The purpose of this paper is to give a proof that the classical James space [5] has the f.p.p., using the beautiful works of Maurey [15] and Lin [12].

Let me point out that in [13], Lin proved positive results concerning the f.p.p. in Banach spaces with unconditional basis. Our paper shows that the ideas arising from Lin's paper are applicable in some Banach spaces with a "good" Schauder basis.

For more detailed history of the f.p.p., we suggest the reader consults [10] and [16] and the references listed therein.

\section{MAIN RESULT}

First recall the definition of the James space $J$. This space consists of sequences $x=\left(x_{n}\right)$ for which $\operatorname{Lim}\left(x_{n}\right)=0$, and $\|x\|_{J}<\infty$ where

$$
\|x\|_{J}=\operatorname{Sup}\left\{\left[\left(x_{p_{1}}-x_{p_{2}}\right)^{2}+\left(x_{p_{2}}-x_{p_{3}}\right)^{2}+\ldots+\left(x_{p_{n-1}}-x_{p_{n}}\right)^{2}+\left(x_{p_{n}}-x_{p_{1}}\right)^{2}\right]^{1 / 2}\right\}
$$

and the supremum is taken over all positive integers $n$ and all increasing sequences of positive integers $\left\{p_{1}, p_{2}, \ldots, p_{n}\right\}$.

Received 8 March 1988

The author wishes to thank J.B. Baillon, G. Godefroy and B. Maurey for useful discussions regarding this paper.

Copyright Clearance Centre, Inc. Serial-fee code: 0004-9729/89 $\$$ A2.00+0.00. 
Remark. Sometimes the term $\left(x_{p_{n}}-x_{p_{1}}\right)$ is dropped, and then we obtain a new space $J_{1}$ which is isomorphic to $J$. In [8] it is proved that any weakly compact convex subset of $J_{1}$ has the normal structure and therefore $J_{1}$ has the f.p.p.

The space $J$ was used to disprove several long-standing conjectures $[14,(\mathrm{I})$ p.25, $103,132],[14,($ II) p.36, 39], $[2,3,4]$ and $[11]$.

For the proof of our result, we need one technical lemma, which seems to be new.

LEMMA 1.

(1) For integers $a \leqslant b$ we denote the interval of integers between $a$ and $b$ by $F$. Consider the natural projection $P_{F}$ associated with the basis of $J$. Then:

$$
\left\|I-P_{F}\right\|^{2} \leqslant 2
$$

(2) Let $u$ and $v$ be defined by:

$$
\begin{gathered}
u=\sum_{a}^{b} \beta_{i} e_{i} \text { and } v=\sum_{c}^{d} \alpha_{i} e_{i} \text { with } a \leqslant b<c-1 \text { and } c \leqslant d, \text { then } \\
\|u+v\| \leqslant \sqrt{2}\|u-v\|
\end{gathered}
$$

ProOF: Since the proof of (1) and (2) uses the same techniques, we give only the proof of (1):

Let $x$ be in $J$ with $\|x\| \leqslant 1$, we have

$$
\left(I-P_{F}\right)(x)=x_{F}=\sum_{i<a} x_{i} e_{i}+\sum_{i>b} x_{i} e_{i}=\sum_{i} y_{i} e_{i}
$$

Let $\left(p_{i}\right)$ denote a strictly increasing finite sequence of integers. There are two • cases:

First case. :

$$
\begin{gathered}
\left\{p_{i}\right\} \cap F=\emptyset \text { then: } \\
\sum_{1}^{n}\left(y_{p_{i}}-y_{p_{i+1}}\right)^{2}+\left(y_{p_{n}}-y_{p_{1}}\right)^{2} \leqslant\|x\|^{2} \leqslant 1
\end{gathered}
$$

Second case. :

$$
\left\{p_{i}\right\} \cap F \neq \emptyset \text { then: }
$$

$$
\begin{aligned}
& \sum_{1}^{n-1}\left(y_{p_{i}}-y_{p_{i+1}}\right)^{2}+\left(y_{p_{n}}-y_{p_{1}}\right)^{2} \\
& \quad=\sum_{i \leqslant j}\left(x_{p_{i}}-x_{p_{i+1}}\right)^{2}+\sum_{i=k}^{i=n-1}\left(x_{p_{i}}-x_{p_{n+1}}\right)^{2}+x_{j}^{2}+x_{k}^{2}+\left(x_{p_{n}}-x_{p_{1}}\right)^{2}
\end{aligned}
$$


with $j \leqslant a \leqslant b \leqslant k$. But:

$$
\begin{aligned}
& \sum_{i=1}^{i=j}\left(x_{p_{i}}-x_{p_{i+1}}\right)^{2}+\sum_{i=k}^{i=n-1}\left(x_{p_{i}}-x_{p_{i+1}}\right)^{2}+\left(x_{p_{n}}-x_{p_{1}}\right)^{2} \\
& \quad \leqslant \sum_{i=1}^{i=j}\left(x_{p_{i}}-x_{p_{i+1}}\right)^{2}+\left(x_{p_{j}}-x_{p_{k}}\right)^{2} \\
& \quad+\sum_{i=k}^{i=n-1}\left(x_{p_{i}}-x_{p_{i+1}}\right)^{2}+\left(x_{p_{n}}-x_{p_{1}}\right)^{2} \leqslant\|x\|^{2} \leqslant 1
\end{aligned}
$$

and $x_{i}^{2}+x_{k}^{2} \leqslant 1$ (because the sequence $\left(x_{n}\right)$ is in $c_{o}$ )

We deduce that:

$$
\left\|x_{f}\right\|^{2} \leqslant 1+1=2 .
$$

Now we state the main theorem.

THEOREM. Every weakly compact convex subset of $J$ has the fixed point property.

Proof: Suppose that there exists a weakly compact, nonempty convex subset $C$ of $J$ and a non-expansive $T: C \rightarrow C$ without fixed point. By Zorn's Lemma $C$ contains a nonempty closed convex subset $K, T$-invariant and minimal with respect to the inclusion. Our hypothesis on $T$ implies that diam $K>0$, without loss of generality we can assume that $\operatorname{diam} K=1$. It is easy to see that $K$ contains a quasifixed sequence $\left(x_{n}\right)$ (that is $\operatorname{Lim}\left\|x_{n}-T\left(x_{n}\right)\right\|=0$ ). Using the fact that $K$ is weakly compact, the sequence $\left(x_{n}\right)$ has a subsequence which is weakly convergent. Since our problem is invariant by translation and by passing to a subsequence, we can assume that $\left(x_{n}\right)$ converges weakly to 0 .

The Karlovitz' Lemma [7] states that for any $x$ in $K$ we have:

$$
\operatorname{Lim}\left\|x_{n}-x\right\|=\operatorname{diam} K=1 .
$$

Since $\left(x_{n}\right)$ converges weakly to 0 and satisfies $(* *)$ then there exists a subsequence $\left(x_{n}^{\prime}\right)$ and a sequence of blocks $\left(u_{n}\right)$ such that:

1) $\operatorname{Lim}\left\|x_{n}^{\prime}-u_{n}\right\|=0$,

2) $\operatorname{Lim}\left\|x_{n+1}^{\prime}-x_{n}^{\prime}\right\|=1$, where $u_{n}=\sum_{i=1_{n}}^{i=b_{n}} \beta_{i}^{n} e_{i}$ with $a_{n}<b_{n}=a_{n+1}$. 
Let $P_{n}$ and $Q_{n}$ denote the natural projections defined by:

$$
P_{n}\left(\sum_{i} \beta_{i} e_{i}\right)=\sum_{i=a_{n}}^{i=b_{n}} \beta_{i} e_{i} \text { and } Q_{n}\left(\sum_{i} \beta_{i} e_{i}\right)=\sum_{i>a_{n+1}} \beta_{i} e_{i}
$$

Then by the construction of $\left(u_{n}\right)$ we have:
i) $\operatorname{Lim}\left\|x \prime_{n}^{\prime}-P_{n}\left(x_{n}^{\prime}\right)\right\|=0$;
ii) $\operatorname{Lim}\left\|x_{n+2}^{\prime}-Q_{n}\left(x_{n+2}^{\prime}\right)\right\|=0$ (because $\left.Q_{n}\left(u_{n+2}\right)=u_{n+2}\right)$;
iii) $\operatorname{Lim}\left\|P_{n}(x)\right\|=\operatorname{Lim}\left\|Q_{n}(x)\right\|=0$ for every $x$ in $J$.

Let $\mathcal{U}$ denote a non-trivial ultrafilter on $\mathbf{N}$. The ultraproduct space $\mathbf{J}$ of $J$ is the quotient space of:

$$
1_{\infty}(J)=\left\{\left(x_{n}\right) ; x_{n} \in J \text { for all } n \in \mathrm{N} \text { and }\left\|\left(x_{n}\right)\right\|_{\infty}=\sup \left\|x_{n}\right\|<\infty\right\}
$$

by $\mathcal{N}=\left\{\left(x_{n}\right) \in 1_{\infty}(J) \operatorname{Lim}_{u}\left\|x_{n}\right\|=0\right\}$. We shall not distringuish between $\left(x_{n}\right) \in$ $1_{\infty}(J)$ and the coset $\left(x_{n}\right)+\mathcal{N} \in \mathbf{J}$. Clearly,

$$
\left\|\left(x_{n}\right)\right\|_{\mathbf{J}}=\operatorname{Lim}_{U}\left\|x_{n}\right\|_{J}
$$

It is also clear that $J$ is isometric to a subspace of $\mathbf{J}$ by the mapping $x \rightarrow(x, x, \ldots)$. Hence, we may assume that $J$ is a subspace of $J$. We will write $\mathbf{x}, \mathbf{y}, \mathbf{z}$ for the general elements of $\mathbf{J}$ and $x, y, z$ for the general element of $J$. In $\mathbf{J}$ we define:

$$
\mathbf{K}=\left\{\mathbf{y} \in \mathbf{J} ; \mathbf{y}=\left(y_{n}\right) \text { with } y_{n} \in K\right\}
$$

and

$$
\mathbf{T}: \mathbf{K} \rightarrow \mathbf{K} \text { with } \mathbf{T}(\mathbf{y})=\mathbf{T}\left(y_{n}\right)=\left(T\left(y_{n}\right)\right)
$$

Clearly $\mathbf{K}$ is a closed convex set with $\operatorname{diam}(\mathbf{K})=\operatorname{diam}(K)=1$, and $\mathbf{T}$ is a nonexpansive map on $\mathbf{K}$. Furthermore, $\mathbf{T}$ has fixed points in $K$. Indeed, if $\left(x_{n}\right)$ is quasi fixed sequence for $T$ in $K$, then $\operatorname{Lim}\left\|x_{n}-T\left(x_{n}\right)\right\|=0$ and hence:

$$
\left\|\mathbf{T}\left(x_{n}\right)-\left(x_{n}\right)\right\|_{\mathbf{J}}=\operatorname{Lim}_{u}\left\|x_{n}-T\left(x_{n}\right)\right\|=0
$$

This means that $\mathbf{T}\left(x_{n}\right)=\left(x_{n}\right)$, that is $\left(x_{n}\right)$ is a fixed point for $\mathbf{T}$ in $\mathbf{J}$. Also, if $\mathbf{T}\left(y_{n}\right)=\left(y_{n}\right)$, then some subsequence of $\left(y_{n}\right)$ is a quasi fixed sequence for $\mathbf{T}$. The Karlovitz Lemma can be stated in the space $\mathbf{J}$ by the following: 
LEMMA 2. [12]: Let $\left(\mathbf{w}_{n}\right)$ be a quasi-fixed sequence for $\mathbf{T}$, then:

$$
\operatorname{Lim}\left\|\mathbf{w}_{n}-x\right\|=\operatorname{diam}(K)=1 \text { for any } x \text { in } K
$$

In other works, if $\mathbf{W}$ is any nonempty closed $\mathbf{T}$-invariant convex subset of $\mathbf{K}$, we have:

$$
\operatorname{Sup}_{w \in \mathbf{W}}\|\mathbf{w}-x\|=\operatorname{diam}(K)=1 \text { for any } x \text { in } K .
$$

Define $x$ and $y$ by: $\mathrm{x}=\left(x_{n}^{\prime}\right)$ and $\mathrm{y}=\left(x_{n+2}^{\prime}\right)$ by ii) we have: $\mathrm{x}=\left(P_{n}\left(x_{n}^{\prime}\right)\right)$ and $\mathbf{y}=\left(Q_{n}\left(x_{n+2}^{\prime}\right)\right)$ and by 2$)$ we have: $\|\mathbf{x}-\mathbf{y}\|=1$.

From Lemma 1, we deduce that:

$$
\|x+y\|^{2} \leqslant 2\|x-y\|^{2}
$$

Let $\mathbf{W}=\left\{\mathbf{w} \in \mathbf{K}, \exists x \in K\right.$ s.t. $\left.\|\mathbf{w}-x\| \leqslant 2^{-1 / 2} \& \operatorname{Sup}(\|\mathbf{w}-\mathbf{x}\|,\|\mathbf{w}-\mathbf{y}\|) \leqslant 1 / 2\right\}$.

Everything was done to ensure that $\frac{x+y}{2}$ is in $W$. Also it is easy to verify that $\mathbf{W}$ is a closed $\mathbf{T}$-invariant convex subset of $\mathbf{K}$. Consider the projections $\mathbf{P}$ and $\mathbf{Q}$ defined on $\mathbf{J}$ by:

$$
\mathbf{P}(\mathbf{z})=\left(P_{n}\left(z_{n}\right)\right) \text { and } \mathbf{Q}(\mathbf{z})=\left(Q_{n}\left(z_{n}\right)\right) \text { where } \mathbf{z}=\left(z_{n}\right)
$$

Since the basis of $\mathbf{J}$ is bimonotone, we have:

$$
\begin{gathered}
\|\mathbf{P}\| \leqslant \operatorname{Sup}\left\|\mathbf{P}_{n}\right\| \leqslant 1 \\
\|\mathbf{Q}\| \leqslant \operatorname{Sup}\left\|\mathbf{Q}_{n}\right\| \leqslant \mathbf{1} \\
\|\mathbf{P}+\mathbf{Q}\| \leqslant \operatorname{Sup}\left\|\mathbf{P}_{n}+\mathbf{Q}_{n}\right\| \leqslant 1 \\
\|\mathbf{I}-\mathbf{Q}\| \leqslant 1
\end{gathered}
$$

Invoking Lemma 1, we have:

$$
\|\mathbf{I}-\mathbf{P}\|^{2} \leqslant 2
$$

Choose $\mathbf{w}$ in $\mathbf{W}$ and $x$ in $K$ such that $\|\mathbf{w}-x\| \leqslant 2^{-1 / 2}$.

One has:

$$
2 \mathbf{w}=(\mathbf{P}+\mathbf{Q})(\mathbf{w})+(\mathbf{I}-\mathbf{P})(\mathbf{w})+(\mathbf{I}-\mathbf{Q})(\mathbf{w})
$$

From the definitions of $\mathbf{P}$ and $\mathbf{Q}$, we can directly derive the following:

$$
\mathbf{P}(x)=\mathbf{Q}(x)=0, \mathbf{P}(\mathbf{x})=\mathbf{x} \text { and } \mathbf{Q}(\mathbf{y})=\mathbf{y}
$$


Using $\left(^{*}\right)$ we deduce that $2 \mathbf{w}=(\mathbf{P}+\mathbf{Q})(\mathbf{w}-x)+(\mathbf{I}-\mathbf{P})(\mathbf{w}-\mathbf{x})+(\mathbf{I}-\mathbf{Q})(\mathbf{w}-\mathbf{y})$. And then we have

$$
2\|\mathbf{w}\| \leqslant\|\mathbf{P}+\mathbf{Q}\|\|\mathbf{w}-x\|+\|\mathbf{I}-\mathbf{P}\|\|\mathbf{w}-\mathbf{x}\|+\|\mathbf{I}-\mathbf{Q}\|\|\mathbf{w}-\mathbf{y}\| .
$$

And using all our previous inequalities, we obtain

$$
2\|w\| \leqslant 2^{-1 / 2}+2^{-1 / 2}+2^{-1} .
$$

This implies

$$
\underset{\mathbf{w}}{\operatorname{Sup}}\|\mathbf{w}\|<1
$$

which yields a contradiction to Lemma 2 .

\section{REFERENCES}

[1] D. Alspach, 'A fixed point free non expansive map', Proc. Amer. Math. Soc. 82 (1981), $423-424$.

[2] A. Andrew, 'James' quasi-reflexive space is not isomorphic to any subspace of its dual', Israel $J$. Math. 38 (1981), 276-282.

[3] S. Bellenot, 'Transfinite duals of quasi-reflexive Banach spaces', Trans. Amer. Math. Soc. 273 (1982), 551-577.

[4] P.G. Casazza, 'James quasi-reflexive space is primary', Israel J. Math. 26 (1977), $294-305$.

[5] R.C. James, 'A non-reflexive Banach space isometric with its second conjugate space', Proc. Nat. Acad. Sci. U.S.A. 37 (1951), 174-177.

[6] R.C. James, 'Banach spaces quasi-reflexive of order one', Studia. Math. 60 (1977), 157-177.

[T] L.A. Karlovitz, 'Existence of fixed points for non-expansive mappings in a space without normal structure', Pacific J. Math. 66 (1976), 153-159.

[8] M.A. Khamsi, 'Normal structure for Banach spaces with Sclauder decomposition' (to appear).

[9] W.A. Kirk, 'A fixed point theorem for mappings which do not increase idstances', Amer. Math. Monthly 72 (1965), 1004-1006.

[10] W.A. Kirk, Fixed point theory for non-expansive mapping $I$, II: Lecture Notes in Math 886, pp. 484-505 (Springer, Berlin, 1981). : Contemp. Math. 18, Pp. 121-140 (A.M.S., Providence RI).

[11] B.L. Lin and R.H. Lohman, 'On generalized James quasi-reflexive Banach spaces', Bull. Inst. Math. Acad. Sinica 8 (1980), 389-399.

[12] P.K. Lin, Texas Functional Analysis Seminar 1982-1983 (The University of Texas Austin).

[13] P.K. Lin, 'Unconditional beses and fixed points of non-expansivre mappings', Pacific J. Math. 116 (1985), 69-76.

[14] J. Lindenstrauss and L. Tzafriri, Classical Banach spaces, Vol I and II (Springer, Berlin-HeidelbergNew York, 1977 and 1979).

[15] B. Maurey, Points fixes des contractions sur un convexe ferme de $L_{1}$ : Seminaire d'analyse fonctionnelle, pp. 80-81 (Ecole Polytechnique, Palaiseau).

[16] S. Reich, 'The fixed point problem for non-expansive mappings, I, II', Amer. Math. Monthly 83 (1976), 266-268. 87, pp. 292-294.

\footnotetext{
University of Southern California

Department of Mathematics DRB 306

1042W.36th Place

Los Angeles, CA 90089-1113

United States of America
} 